\title{
Morfologia matemática na extração de bordas de uma imagem IKONOS-2 RGB fusionada
}

\author{
Edges extraction of fusion IKONOS-2 RGB image with mathematical morphology
}

\author{
Ana Lúcia Bezerra Candeias ${ }^{1}$ \\ João Rodrigues Tavares Junior ${ }^{1}$ \\ Priscilla Heliênay Oliveira do Nascimento ${ }^{1}$ \\ Carla Julliane Marques de Moura ${ }^{1}$ \\ Erivaldo Antônio da Silva²
}

\begin{abstract}
${ }^{1}$ Departamento de Engenharia Cartográfica, Centro de Tecnologia e Geociências - CTG, Universidade Federal de Pernambuco - UFPE, Av. Prof. Moraes Rego, 1235 - Cidade Universitária, Cep 50670-901, Recife, PE, Brasil. ${ }^{2}$ Departamento de Cartografia, Faculdade de Ciências e Tecnologia, Universidade Estadual Paulista "Júlio de Mesquita Filho" - UNESP, Presidente Prudente - SP, Brasil.
\end{abstract}

analucia@ufpe.br, joaoufpe@gmail.com, priscillahelienay@ hotmail.com, carlaju_marques@hotmail.com, silva.erivaldo@gmail.com

Recebido em 07/07/2015 - Aceito em 21/03/2016

Received on 07/07/2015 - Accepted on 03/21/2016

\begin{abstract}
RESUMO - As ferramentas da Morfologia Matemática são utilizadas neste artigo para extração de bordas externas em imagens de alta resolução. A área teste foi um recorte de uma imagem fusionada do satélite IKONOS-2, contendo o Lago do Cavouco, no entorno do Campus Joaquim Amazonas da Universidade Federal de Pernambuco (UFPE). Para obter a borda externa do lago, utilizou-se a implementação de dilatação e operações aritméticas no software SPRING. A partir da imagem RGB fusionada, extraiu-se as bordas de cada uma das bandas dessa imagem, também se extraiu a borda obtida da componente 'I' da transformação RGB para IHS. Além disso, extraiu-se as bordas de 'PC1' obtida da transformação RGB para Principais Componentes. Obteve-se os histogramas, momentos, diagrama de dispersão e um perfil das imagens de borda para se comparar quantitativamente os métodos. Observou-se que a 'PC1' gerou bordas com maior contraste (maior desvio padrão e variância) e menor correlação com as demais bordas obtidas. Isto indicou que a extração de bordas a partir de PC1 apresentou melhor resultado. Ressalta-se que o método de extração de bordas é geral e não utiliza limiares na definição de bordas.
\end{abstract}

Palavras-chave: borda de imagem colorida, processamento de imagem, sensoriamento remoto.

ABSTRACT - Mathematical Morphology tools are used in this article to extract edges in high-resolution images. The test area was part of a merged image of IKONOS-2 satellite, containing Cavouco's lake, surrounding the Campus Joaquim Amazonas Federal University of Pernambuco (UFPE) was used. For the outer edge of the lake, we used the implementation of dilation and arithmetic operations in the SPRING software. It was extracted edges of each band of the merged color image, and also it used the edge extraction from component "I" of transforming RGB to HSI. Likewise, It was extracted the edges of 'PC1' obtained the transformation RGB to Principal Components. Results of edge extraction methods were compared by using histograms, moments, scatter diagram, and profile. It was observed that the 'PC1' generated edges with higher contrast (higher standard deviation and variance), and lower correlation with the other edges obtained. This indicated that the extraction of edges from PC1 showed better results. Furthermore, the edge extraction method showed here is general and does not use thresholds in the edges definition.

Keywords: edge color image, image processing, remote sensing.

\section{INTRODUÇÃO}

A Morfologia Matemática (MM) é um método não linear do processamento de imagens, desenvolvido nos anos 1960 pelos pesquisadores Matheron e Serra na École Nationale Supérieure des Mines de Paris (Serra, 1982). No Brasil, autores como: Silva (1995), Candeias (1997), Banon e Barrera (1998), Ishikawa et al (2010), Rodrigues et al. (2010), Candeias et. al. (2013) desenvolveram pesquisas em MM.
Os operadores básicos são a erosão e a dilatação da imagem $f$ por um elemento estruturante B. Essa imagem $f$ pode ser colorida, em níveis de cinza, ou binária.

Candeias e Sousa (1996), Ângulo e Serra (2003), Evans e Liu (2006), Ângulo e Serra (2007), Li e Xiaoming, L. (2010) desenvolveram pesquisas sobre a morfologia matemática para imagens coloridas. Este tipo de abordagem utiliza, de forma geral, a decomposição de bandas do espaço RGB ou transformação do espaço de cores, na análise das bordas. Em Gonzalez e Woods 
(2000) tem-se a definição do modelo de cores RGB e a transformação desse espaço em outros tais como o IHS.

As imagens de sensoriamento remoto auxiliam na espacialização de estudos do ambiente terrestre (Novo, 2010; Schowengerdt, 2007) e a extração de bordas nessas imagens pode ser obtida usando MM (Candeias, 1997). $\mathrm{Na}$ extração de borda de um lago, é importante observar a resposta espectral para água e para os outros alvos que o cercam. No caso aqui estudado tem-se uma imagem com apenas bandas na faixa do visível. A água sem sedimentos, possui na faixa do vermelho, menor resposta espectral que na faixa do azul ou na faixa do verde (Novo, 2010) e, portanto, na banda do vermelho, haverá maior contraste da água com solo, vegetação e áreas construídas pois possuem resposta espectral mais alta.

Neste artigo analisa-se a extração das bordas usando MM de uma imagem colorida de alta resolução fusionada do IKONOS-2. Obtém-se também a extração de bordas para a componente I da transformação IHS e das Principais Componentes usando a primeira componente (PC1). Tanto a componente I, como a primeira componente (PC1) possuem em sua representação um mapeamento de uma imagem RGB para uma imagem monocromática. As bordas extraídas de I e de PC1, portanto, sintetizam bordas de uma imagem colorida.
O desempenho da extração de bordas foi avaliado para cada uma das bandas da composição RGB, para a componente I e para a componente PC1.

\subsection{Operadores Básicos da Morfologia Matemática}

O efeito de dilatação e da erosão sobre uma imagem binária $X$ utilizando o elemento estruturante B (quadrado $3 \times 3)$ é mostrado na Figura 1. A dilatação $\delta_{B}(X)$ avalia máximos locais, enquanto a erosão $\left(\varepsilon_{B}(X)\right)$, mínimos locais. Supondo que $X$ seja uma imagem em níveis de cinza, tem-se como efeito da dilatação uma imagem borrada e mais clara e na erosão uma imagem borrada e mais escura, como é mostrado a Figura 2.
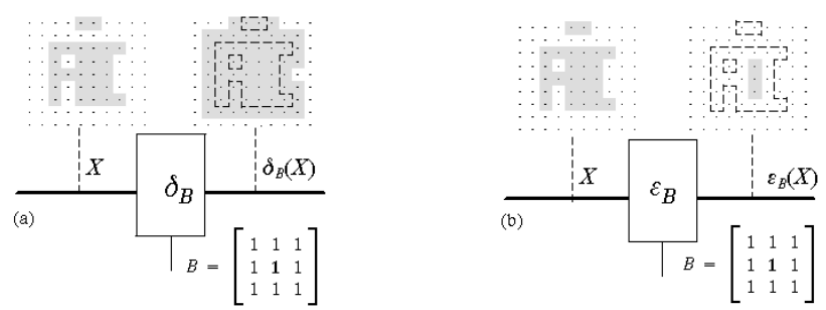

Figura 1 - Operadores Básicos da $\mathrm{MM}$ e a imagem binária $X$. (a) Dilatação $\delta_{B}(X)$. (b) Erosão $\varepsilon_{B}(X)$

Fonte: Banon e Barrera (1998).

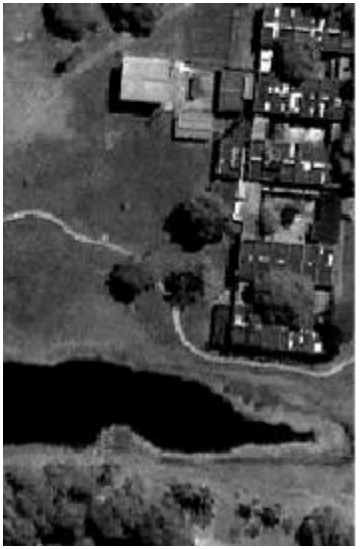

(a)

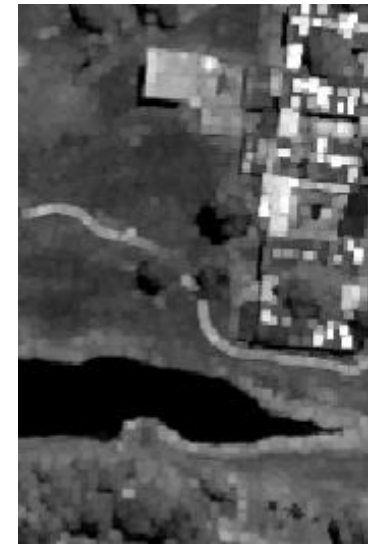

(b)

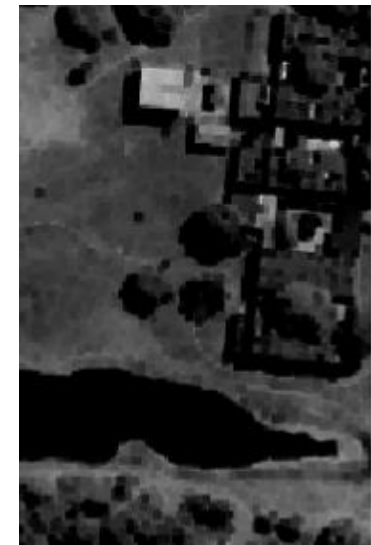

(c)

Figura 2 - Operadores Básicos da MM sobre a imagem em níveis de cinza $X$.

(a) Imagem em níveis de cinza $X$. (b) Dilatação $\delta_{B}(X)$. (c) Erosão $\varepsilon_{B}(X)$.

Para o caso de uma imagem $X$ binária ou em níveis de cinza, tem-se na MM a definição de três tipos de borda: externa, interna e gradiente. $\mathrm{O}$ elemento estruturante irá definir a largura dessa borda (CANDEIAS et al., 2013; CANDEIAS, 1997).

Pode-se avaliar a borda colorida supondo a aplicação da transformação para cada banda ou usando uma transformação que resuma a composição RGB em uma única banda. Nesse trabalho, utiliza-se a componente I (intensidade) da transformação IHS e PC1(primeira componente principal) da transformação de Principais Componentes para avaliação de uma borda colorida.

A seguir tem-se a definição da borda interna, externa e gradiente. Será usado no trabalho a borda externa por segmentar o lago do seu lado externo.
Maiores detalhes ver em (CANDEIAS et al, 2013). As Equações (1), (2) e (3) mostram matematicamente estas bordas.

a) Borda interna

A borda interna de $X$ supondo um elemento estruturante $B$ é definida por:

$$
f=X-\varepsilon_{B}(X)
$$

b) Borda externa

A borda externa de $X$ supondo um elemento estruturante $B$ é definida por

$$
f=\delta_{B}(X)-X
$$


c) Gradiente

O gradiente de $X$ supondo um elemento estruturante $B$ é definido por

$$
f=\delta_{B}(X)-\varepsilon_{B}(X) .
$$

\subsection{Transformação IHS}

O modelo de cores RGB pode ser mapeado para o modelo IHS e vice-versa. A intensidade é denotada por I (intensity), o matiz por $\mathrm{H}$ (hue) e a saturação por $\mathrm{S}$ (saturation). A Figura 3 apresenta uma representação gráfica dessa transformação. Em Gonzalez e Woods (2000) tem-se um detalhamento sobre a transformação IHS e suas equações. A componente I é definida como a média das bandas $R, G$ e $B$ e pode ser usada quando se deseja observar as bordas de uma imagem colorida pois faz uma simplificação do mapeamento de três bandas em uma imagem de níveis de cinza.

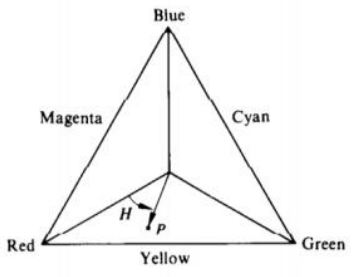

(a)

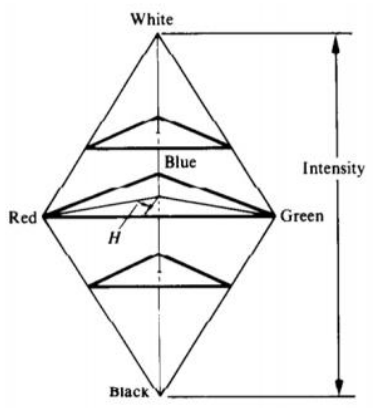

Figura 3 - Representação da modelo IHS.

Fonte: Gonzalez e Woods (2000).

\subsection{Principais Componentes}

A técnica de Principais Componentes (PC), transforma dados multivariados com variáveis correlacionadas, em variáveis não correlacionadas supondo que essas variáveis não correlacionadas são combinações lineares dos dados originais. Supondo uma imagem com $n$ bandas originais $\left(B_{1}, B_{2}, \ldots, B_{\mathrm{n}}\right)$, obtém-se
$\mathrm{PC}_{1}, \mathrm{PC}_{2}, \ldots, \mathrm{PC}_{\mathrm{n}}$ como uma combinação linear de $B_{i}$, onde $i=1, \ldots, n$. E $n$ é o número de bandas multiespectrais.

Dentre as aplicações da técnica de PC tem-se a compressão de informação nas primeiras componentes e também em fusão de imagens que são usadas neste trabalho. Maiores detalhes desta técnica podem ser encontrados em Schowengerdt (2007) e Fonseca et al. (2011).

A imagem colorida fusionada foi obtida aqui usando as bandas multiespectrais 321 e a banda pancromática do IKONOS-2. O resultado é uma imagem com resolução espacial da pancromática e espectral das bandas 321 .

A componente PC1 da imagem fusionada concentra a maior quantidade de informação da entrada da imagem. Ela é usada neste trabalho para avaliar a borda externa do Lago do Cavouco.

\section{METODOLOGIA DO TRABALHO}

A metodologia foi dividida nas seguintes etapas:

a) Seleção da área teste, onde foi usado um recorte de uma imagem IKONOS fusionada $f_{1}$ (pancromática + bandas 1, 2 e 3) com data de aquisição de 15/04/2013, 12:46 GMT. A área selecionada faz parte do campus da UFPE (Figura 4). A fusão foi feita usando Principais Componentes.

b) As bordas externas adquiridas por $\mathrm{MM}$ foram obtidas para cada banda da imagem $f_{1}$ e gerando respectivamente as imagens em níveis de cinza $f_{2}, f_{3}$ e $f_{4}$.

c) Aplicou-se a transformação RGB para IHS da imagem $f_{1}$, e utilizou-se a componente I para se obter a borda externa denotada de $f_{5}$.

d) Aplicou-se a transformação RGB para Principais Componentes da imagem $f_{1}$, e utiliza-se a componente PC1 para obter a borda externa denotada de $f_{6}$.

e) Comparou-se os métodos com diagrama de dispersão e com um perfil das imagens de borda. 


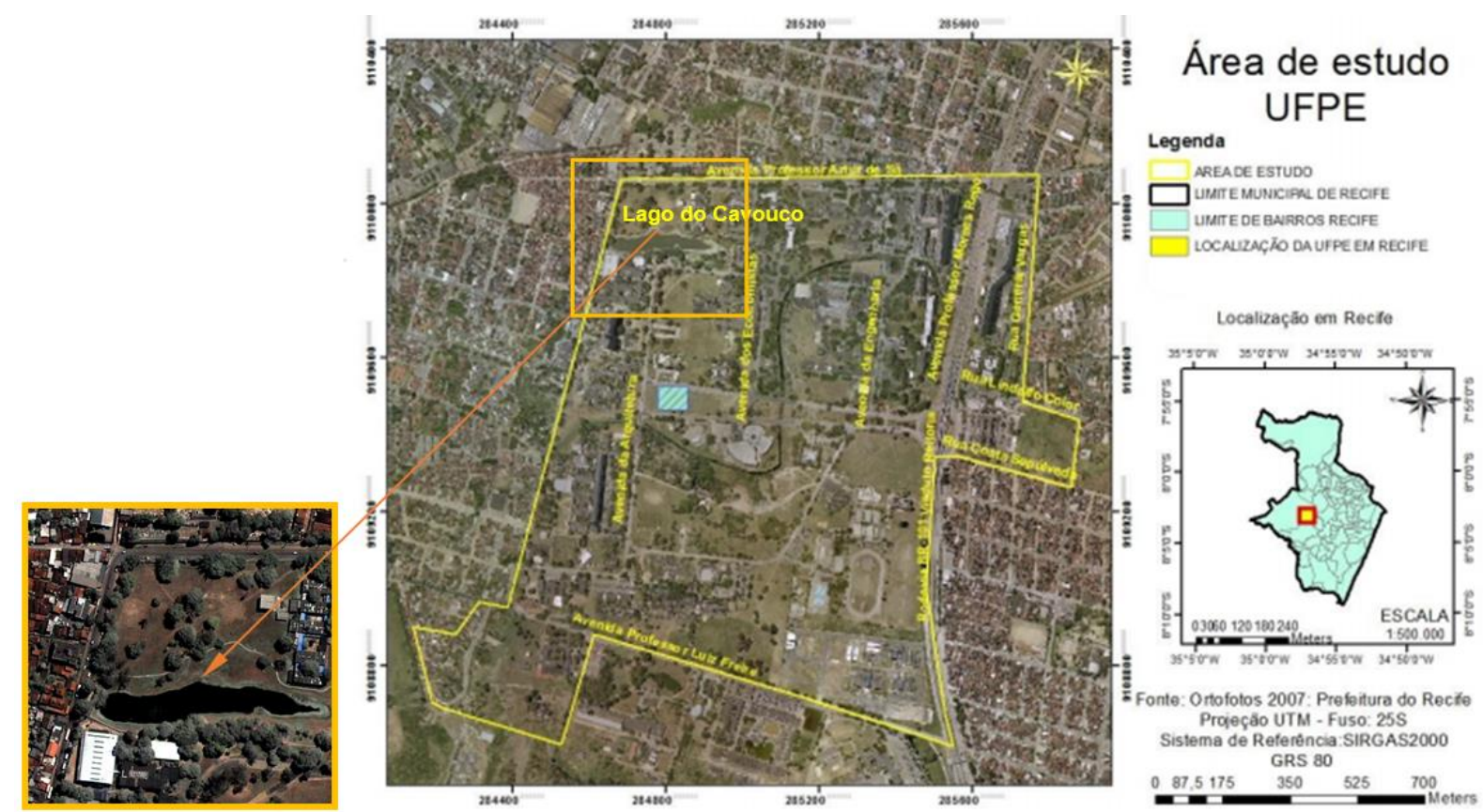

Figura 4 - Localização da área com o recorte da imagem IKONOS-2 do Campus Joaquim Amazonas da UFPE com data de aquisição de 15/04/2013. Adaptada: Silva (2014).

Na implementação da metodologia utilizou-se o software SPRING 5.3 desenvolvido pelo Instituto Nacional de Pesquisas Espaciais (INPE). A Tabela 1 mostra alguns parâmetros do IKONOS-2.

Tabela 1 - Alguns parâmetros do IKONOS-2

\begin{tabular}{c|c}
\hline Parâmetro & Valor \\
\hline $\begin{array}{c}\text { Resolução espectral da banda } \\
\text { pancromática (PAN) }(\mu \mathrm{m})\end{array}$ & $0,45-0,90 \mu \mathrm{m}$ \\
\hline & $0,45-0,53 \mu \mathrm{m}$ \\
$($ azul $)$ & $0,52-0,61 \mu \mathrm{m}$ \\
(verde) & $0,64-0,72 \mu \mathrm{m}$ \\
Resolução espectral das & $($ vermelho $)$ \\
bandas multiespectrais (MS) & $0,76-0,86 \mu \mathrm{m}$ \\
$(\mu \mathrm{m})$ & (infravermelho próximo) \\
\hline Resolução espacial & $1 \mathrm{~m}$ PAN $(0,82 \mathrm{~m}$ no nadir), \\
& 4 m MS (3,2 m no nadir) \\
\hline Quantização do pixel & 11 bit \\
\hline
\end{tabular}

Adaptado: eoPortal Directory (2014)

\section{RESULTADOS}

Apresenta-se aqui três (3) resultados para a borda obtidas pela MM e que são:

a) Borda externa obtida de cada banda ( $\mathrm{R}, \mathrm{G}$ e B) de uma imagem colorida $f_{1}$ Equação (1), (2) e (3) da Seção 11.

b) Borda da Componente I (transformações IHS de uma imagem colorida $f_{1}$ - Equação (4)) e onde I teoricamente, é dada por uma média das bandas R, G e B originais (Gonzalez e Woods, 2000).

c) Borda da primeira componente da imagem colorida $f_{1}$ ((Principais Componentes - Equação (5)) e onde $\mathrm{PC}_{1}$, teoricamente, é dada por uma combinação linear das bandas $\mathrm{R}, \mathrm{G}$ e $\mathrm{B}$ (Schowengerdt, 2007; Fonseca et al., 2011).

Para o caso (a) são obtidas três bordas, uma para cada imagem monocromática da imagem colorida $f_{1}\left(f_{2}, f_{3}\right.$ e $f_{4}$ ).

Já para os casos (b) e (c) tem-se transformações onde a borda de uma imagem colorida $f_{1}$ é obtida a partir de uma única imagem em níveis de cinza $\left(f_{5}\right.$ ou $\left.f_{6}\right)$.

\subsection{Bordas externas adquiridas por $\mathrm{MM}$}

Para extrair a borda externa da imagem fusionada RGB utilizou-se as seguintes equações:

$$
\begin{aligned}
& f_{2}=\delta_{B}\left(f_{1, \mathrm{R}}\right)-f_{1, \mathrm{R}} \\
& f_{3}=\delta_{B}\left(f_{1, \mathrm{G}}\right)-f_{1, \mathrm{G}} \\
& f_{4}=\delta_{B}\left(f_{1, \mathrm{~B}}\right)-f_{1, \mathrm{~B}}
\end{aligned}
$$

Onde $f_{1}, \mathrm{R}, f_{1}$, G e $f_{1}$, B são, respectivamente, as bandas $\mathrm{R}$, $\mathrm{G}$, e B da imagem fusionada $f_{1}$.

A Figura 5(b) apresenta uma composição colorida dos resultados obtidos das Equações (1), (2) e (3). Observa-se que existem bordas mais claras (bordas fortes) e mais escuras (bordas fracas). Gonzalez e Woods (2000) descrevem o cubo das cores e que representa aqui todas as cores possíveis para a borda externa no intervalo [0 max]. 
Nas Figuras 5(b) e 5(c) observam-se que as cores predominantes estão em tons de cinza e, portanto, representadas na diagonal principal do cubo das cores (Figura 5(d)) e que mapeia de preto a branco. Algumas bordas das Figuras 5(b) e 5(c), porém, são mais azuladas ou avermelhadas e estão representadas fora da diagonal principal do cubo (Figura 5(d)). A Tabela 2 mostra os pontos extremos do cubo e as respectivas cores do intervalo [0 $\operatorname{lax}]$. O valor de $\max$ depende do sensor utilizado e é dado por $\max =2^{\text {Quantização do pixel }}-1$. No caso do IKONOS-2 tem-se $\max =2^{11}-1$, e, portanto, $\max =$ 2047.

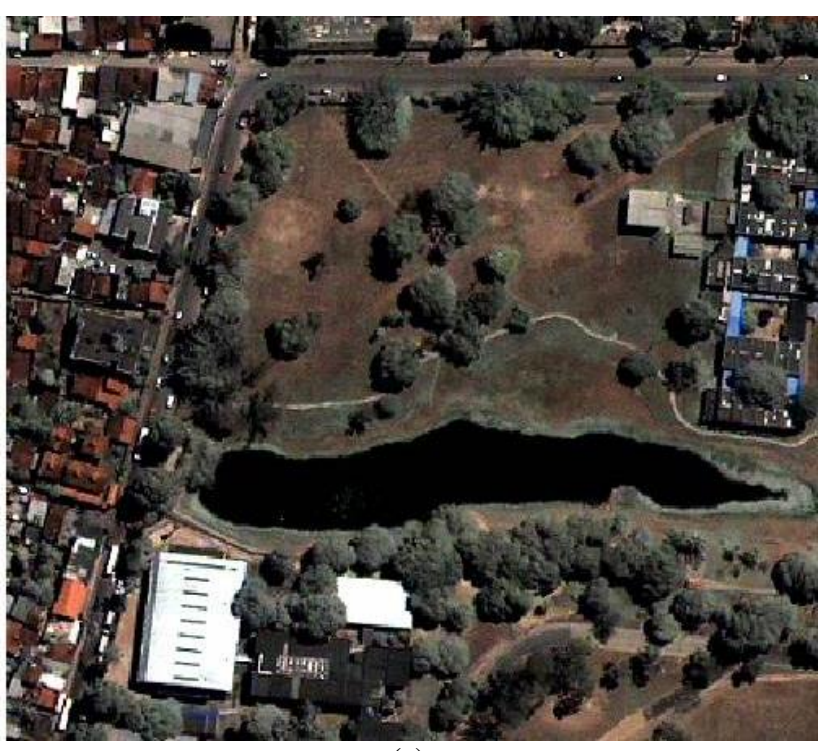

(a)

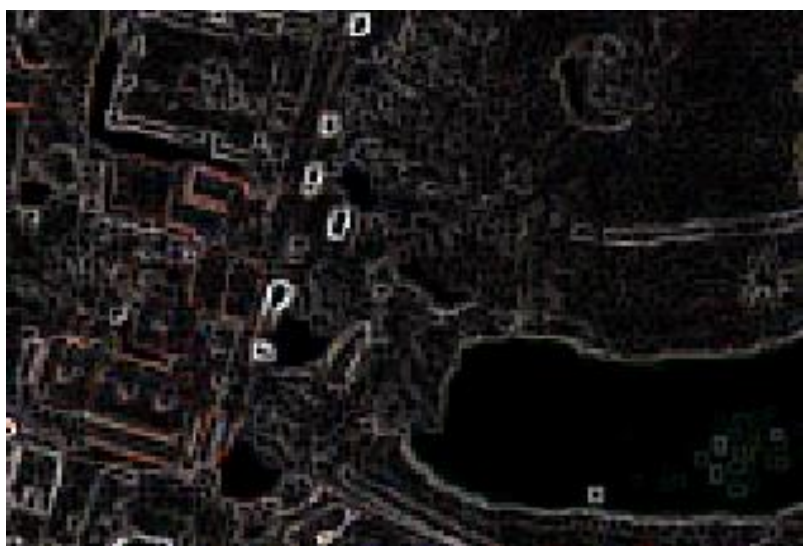

(c)
Tabela 2 - Cores dos pontos extremos do cubo das cores para a composição colorida $\operatorname{RGB} f_{2}, f_{3}, f_{4}$

\begin{tabular}{c|c|c|c}
\hline $\mathbf{R}=\boldsymbol{f}_{\mathbf{4}}$ & $\mathbf{G}=\boldsymbol{f}_{\mathbf{3}}$ & $\mathbf{B}=\boldsymbol{f}_{\mathbf{2}}$ & $\mathbf{C o r}$ \\
\hline 0 & 0 & 0 & Preto \\
\hline 0 & 0 & $\max$ & Azul \\
\hline 0 & $\max$ & 0 & Verde \\
\hline 0 & $\max$ & $\max$ & Ciano \\
\hline $\max$ & 0 & 0 & Vermelho \\
\hline $\max$ & 0 & $\max$ & Magenta \\
\hline $\max$ & $\max$ & 0 & Amarelo \\
\hline $\max$ & $\max$ & $\max$ & Branco \\
\hline
\end{tabular}

A Figura 6 exibe os histogramas das bordas externas supondo que $\max =255$. As Tabelas 3,4 e 5 apresentam respectivamente as estatísticas, matriz de covariância e de correlação das bordas externas $f_{2}, f_{3}$ e $f_{4}$

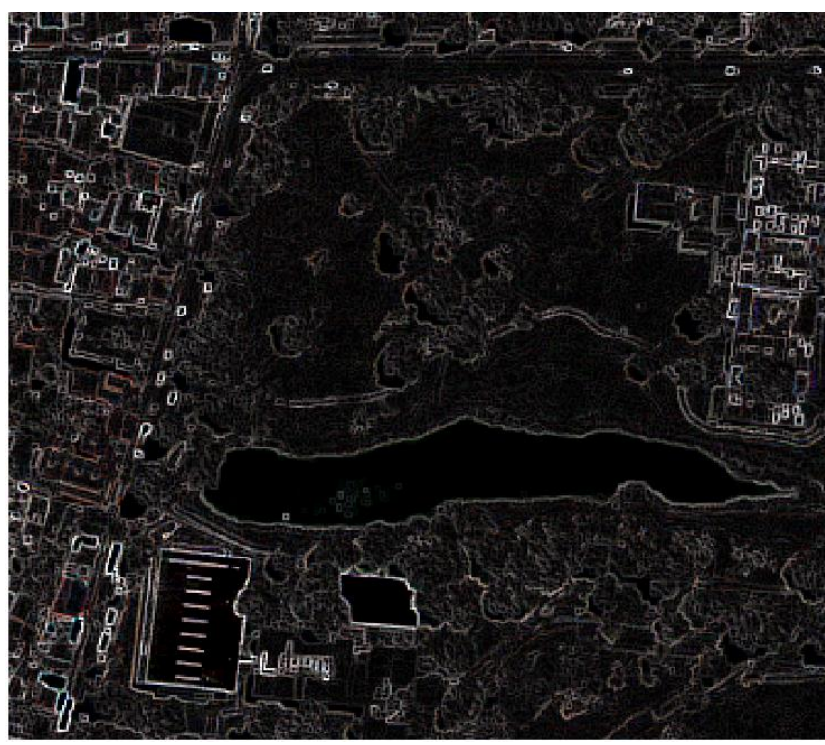

(b)

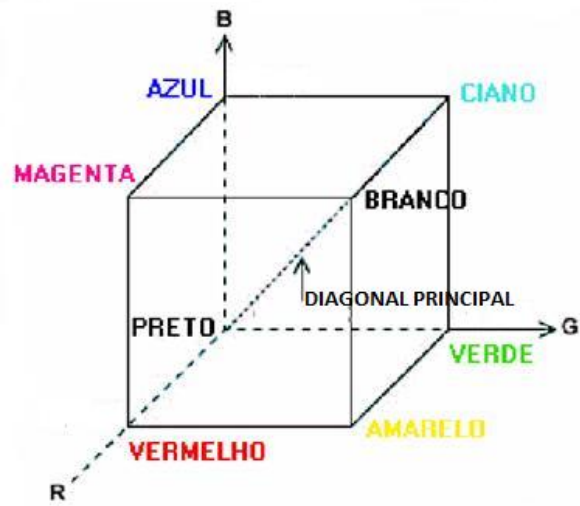

(d)

Figura 5 - Lago do Cavouco. (a) Imagem Fusionada RGB IKONOS-2. (b) Composição RGB $\left(f_{4} f_{3} f_{2}\right)$ bordas externas. (c) Ampliação de parte da Composição RGB $\left(f_{4} f_{3} f_{2}\right)$ bordas externas. Cubo das cores da Composição RGB $\left(f_{4} f_{3} f_{2}\right)$.

\subsection{Borda externa da transformação RGB para IHS}

Foi utilizada a transformação IHS para reunir as informações RGB da imagem fusionada $f_{1}$ e então, a transformação de borda externa, foi aplicada a componente I, gerando o resultado denotado de $\mathrm{f}_{5}$. A componente I é a média das bandas R, G e B. 


$$
f_{5}=\delta_{B}\left(f_{1, \mathrm{I}}\right)-f_{1, \mathrm{I}}
$$

Onde $f_{1}$, I é a componente I da transformação IHS a imagem fusionada $f_{1}$.

\subsection{Borda externa da transformação RGB para Principais Componentes}

Foi utilizada a transformação de Principais Componentes para reunir as informações RGB da imagem fusionada $f_{1}$, a transformação de borda externa, foi aplicada a componente PC1, gerando o resultado denotado de $\mathrm{f}_{6}$. PC1 é dada por uma combinação linear das bandas fusionadas.

$$
f_{6}=\delta_{B}\left(f_{1, \mathrm{PC} 1}\right)-f_{1, \mathrm{PC} 1}
$$

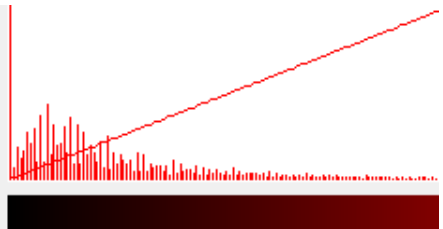

(a) Histograma de $f_{2}$

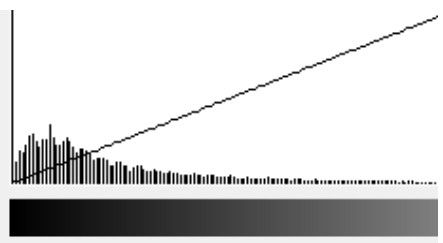

(d) Histograma de $f_{5}$
Onde $f_{1}, \mathbf{P C} 1$ e a primeira componente (PC1) das Principais Componentes da imagem fusionada $f_{1}$.

\subsection{Análise comparativa dos resultados}

A Figura 6 apresenta os histogramas de $f_{2}, f_{3}, f_{4}$ $f_{5}$ e $f_{6}$. O histograma com maior diferença entre os demais é o $f_{6}$. Os outros histogramas são parecidos, isto é, representam imagens similares. Essa similaridade pode ser vista também pela Tabela 3 que mostra os momentos, a matriz de covariância (Tabela 4) e as correlações (Tabela 5) que estão próximos entre si para $f_{2}, f_{3}, f_{4}$ e $f_{5}$ já $f_{6}$ mostra maior diferença.

A imagem $f_{5}$ é uma média aritmética entre $f_{2}, f_{3}$, $f_{4}$, e isto se reflete no cálculo apresentado dos momentos, a matriz de covariância e correlação para essa imagem.

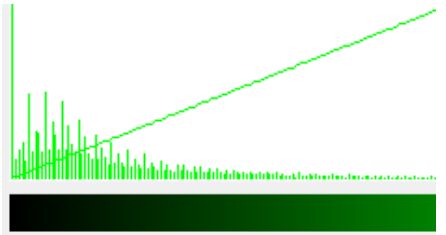

(b) Histograma de $f_{3}$

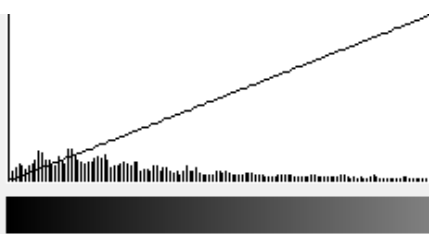

(e) Histograma de $f_{6}$

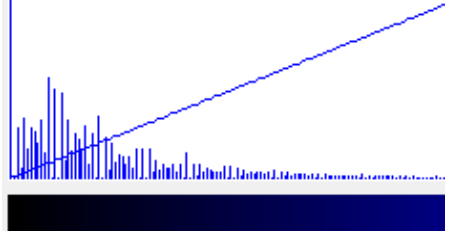

(c) Histograma de $f_{4}$

Figura 6 - Histogramas das bordas externas $f_{2}, f_{3}, f_{4}, f_{5}$ e $f_{6}$.

Tabela 3 - Momentos calculados das bordas externas $f_{2}, f_{3}, f_{4,} f_{5}$ e $f_{6}$.

\begin{tabular}{c|r|r|r|r|r}
\hline Banda & Média & Desvio Padrão & Variância & 3_Momento & 4_Momento \\
\hline$f_{2}$ & 28,9662 & 34,5046 & $1.190,57$ & $98.318,3$ & $1,52765 \mathrm{e}+07$ \\
\hline$f_{3}$ & 26,6588 & 32,2142 & $1.037,76$ & $84.783,6$ & $1,28879 \mathrm{e}+07$ \\
\hline$f_{4}$ & 26,8566 & 32,3547 & $1.046,83$ & $86.773,7$ & $1,33738 \mathrm{e}+07$ \\
\hline$f_{5}$ & 26,7499 & 33,1641 & $1.099,86$ & $92.527,4$ & $1,42571 \mathrm{e}+07$ \\
\hline$f_{6}$ & 40,3992 & 45,2694 & $2.049,32$ & 161.587 & $2,64844 \mathrm{e}+07$ \\
\hline
\end{tabular}

Tabela 4 - Matriz de Covariância das bordas externas $f_{2}, f_{3}, f_{4}, f_{5}$ e $f_{6}$.

\begin{tabular}{rrrrrr}
$f_{2}$ & $1.190,57$ & $1.088,49$ & $1.079,09$ & $1.063,33$ & $1.387,82$ \\
$f_{3}$ & $1.088,49$ & $1.037,76$ & $1.026,66$ & 995,812 & $1.279,65$ \\
$f_{4}$ & $1.079,09$ & $1.026,66$ & $1.046,83$ & 996,497 & $1.265,37$ \\
$f_{5}$ & $1.063,33$ & 995,81 & 996,45 & $1.099,86$ & $1.246,51$ \\
$f_{6}$ & $1.387,82$ & $1.279,65$ & $1.265,37$ & $1.246,51$ & $2.049,32$ \\
\hline
\end{tabular}


Tabela 5 - Matriz de Correlação das bordas externas $f_{2}, f_{3}, f_{4}, f_{5}$ e $f_{6}$.

\begin{tabular}{cccccc}
\hline & $f_{2}$ & $f_{3}$ & $f_{4}$ & $f_{5}$ & $f_{6}$ \\
$f_{2}$ & 1,000000 & 0,979262 & 0,966587 & 0,929230 & 0,88849 \\
$f_{3}$ & 0,979262 & 1,000000 & 0,985011 & 0,932097 & 0,877481 \\
$f_{4}$ & 0,966587 & 0,985011 & 1,000000 & 0,928688 & 0,863923 \\
$f_{5}$ & 0,929230 & 0,932097 & 0,928688 & 1,000000 & 0,830274 \\
$f_{6}$ & 0,888490 & 0,877481 & 0,863923 & 0,830274 & 1,000000 \\
\hline
\end{tabular}

Os diagramas de dispersão estão mostrados na Figura 7 para as bandas $f_{2}, f_{3}, f_{4}, f_{5}$ e $f_{6}$. O resultado com menor dispersão foi de $f_{4}$ versus $f_{3}$, e a maior dispersão foi de $f_{6}$ versus $f_{5}$. Pela Tabela 5 observa-se que $f_{4}$ e $f_{3}$ são as bandas mais correlacionadas, enquanto $f_{6}$ e $f_{5}$ são as menos correlacionadas. A vantagem de usar o diagrama de dispersão é ter uma visualização do comportamento para cada nível de cinza.

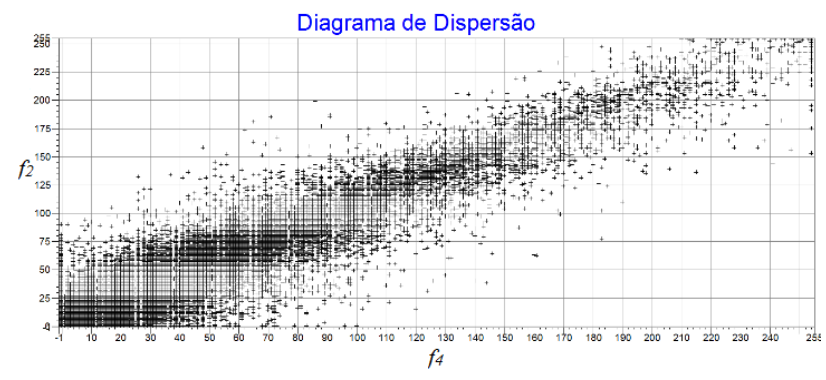

(a) Diagrama de dispersão de $f_{2}$ versus $f_{4}$

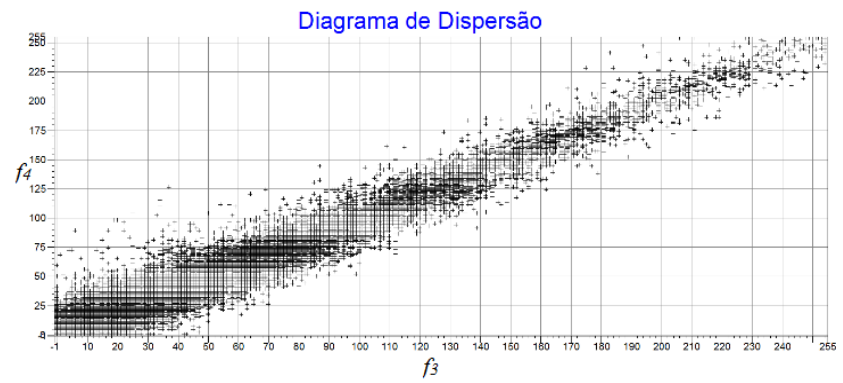

(c) Diagrama de dispersão de $f_{4}$ versus $f_{3}$

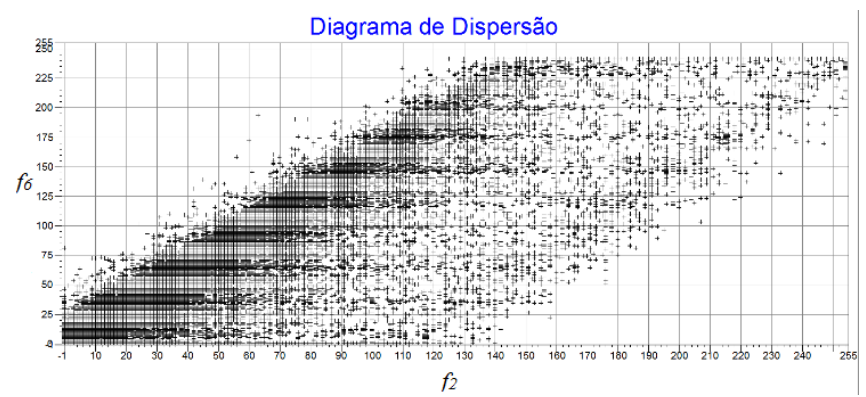

(e) Diagrama de dispersão de $f_{6}$ versus $f_{2}$

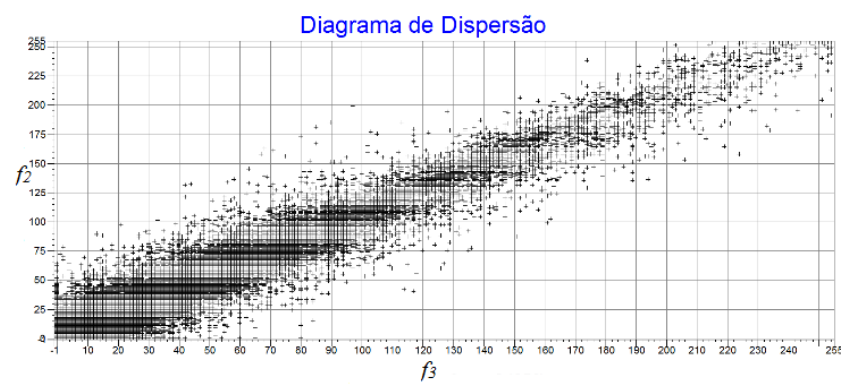

(b) Diagrama de dispersão de $f_{2}$ versus $f_{3}$

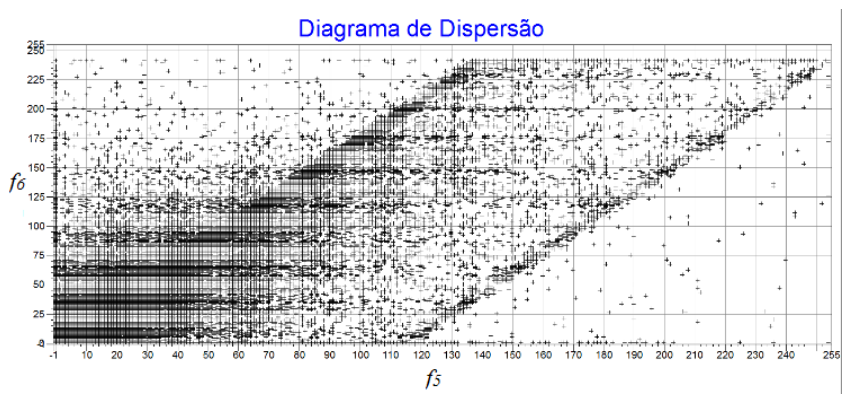

(d) Diagrama de dispersão de $f_{6}$ versus $f_{5}$

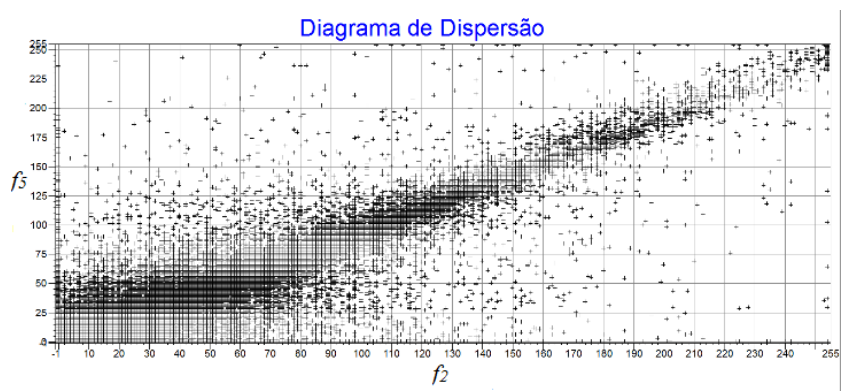

(f) Diagrama de dispersão de $f_{5}$ versus $f_{2}$

Figura 7 - Diagramas de dispersão das bandas $f_{2}, f_{3}, f_{4}, f_{5}$ e $f_{6}$.

Foi extraído o perfil vertical na coluna: 159, entre as linhas: 236 e 257 (Figura 8) para as imagens $f_{2}, f_{3}, f_{4}, f_{5}$ e $f_{6}$. A Figura 9 mostra o gráfico desses perfis. Para $f_{2}, f_{3}, f_{4}$ e para $f_{5}$, observam-se perfis semelhantes e isto pode ser verificado também pela alta correlação dos resultados obtidos (Tabela 5). Já para $f_{6}$ tem-se valores mais altos de níveis de cinza (Figura 9) para as bordas. Tem-se também que $f_{6}$ possui desvio padrão, variância e covariância altas quando comparada aos demais resultados de $f_{2}, f_{3}, f_{4}$ e $f_{5}$ (Tabela 3 e Tabela 4). Ou ainda analisando a Tabela 5, 
apresenta valores de correlação baixo quando comparados $\operatorname{com} f_{2}, f_{3}, f_{4}$ e $f_{5}$ mostrando que $f_{6}$.

A Figura 10(b) apresenta a combinação colorida $f_{1, R}$, $f_{2, \mathrm{G}}, f_{6}$. Já a Figura $10(\mathrm{c})$ mostra $f_{6}$. São extraídas todas as bordas em $f_{6}$, pois não foi imposto um limiar. Nota-se que são extraídas também as sombras dos objetos.

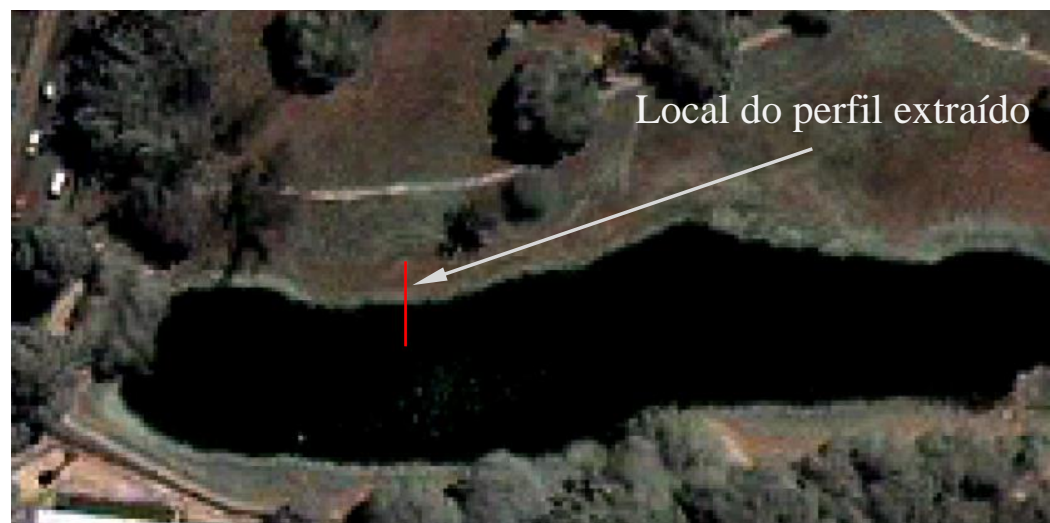

Figura 8 - Visualização do Perfil vertical da coluna: 159, entre as linhas: 236 e 257 em $f_{1}$.

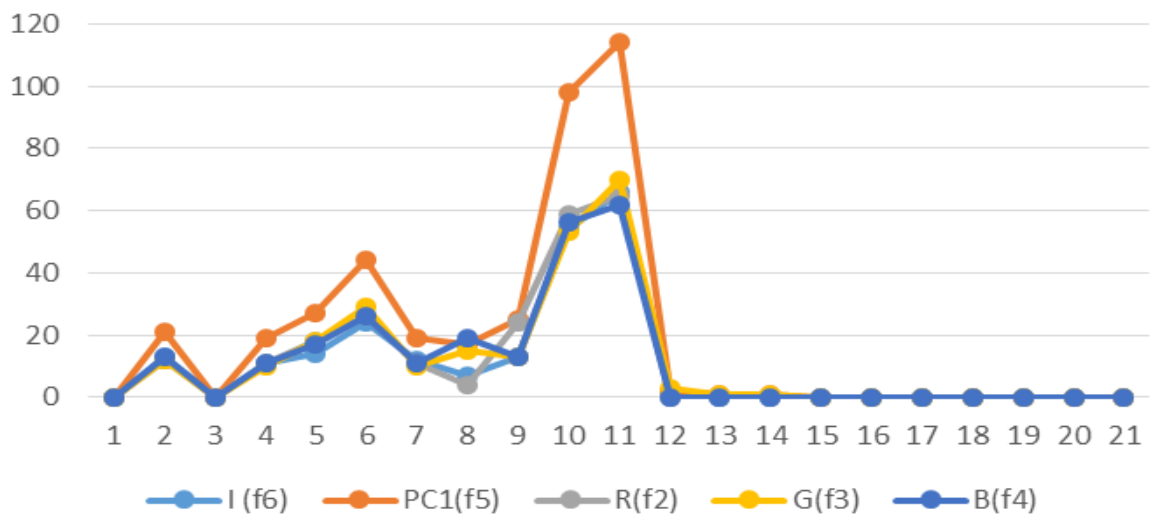

Figura 9 - Perfil de $f_{2}, f_{3}, f_{4}, f_{5}$ e $f_{6}$

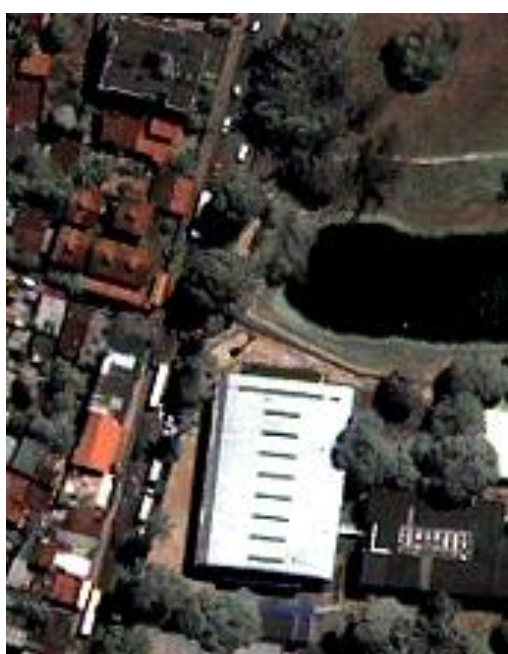

(a) Imagem colorida $f_{1}$

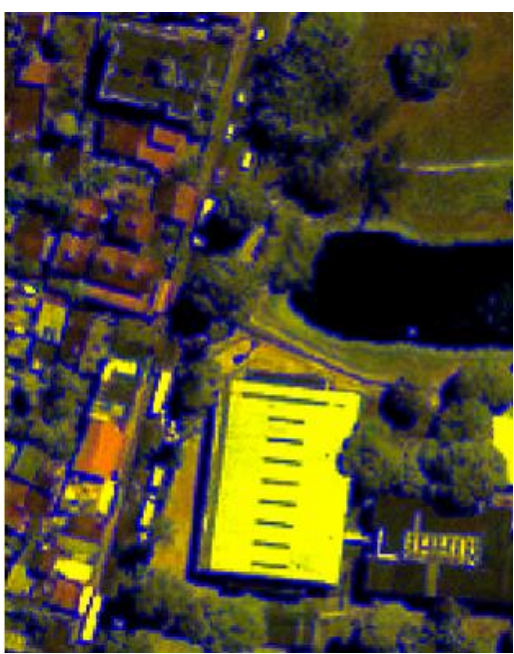

(b) Composição RGB $f_{1, \mathrm{R}}, f_{2, \mathrm{G}}, f_{6}$

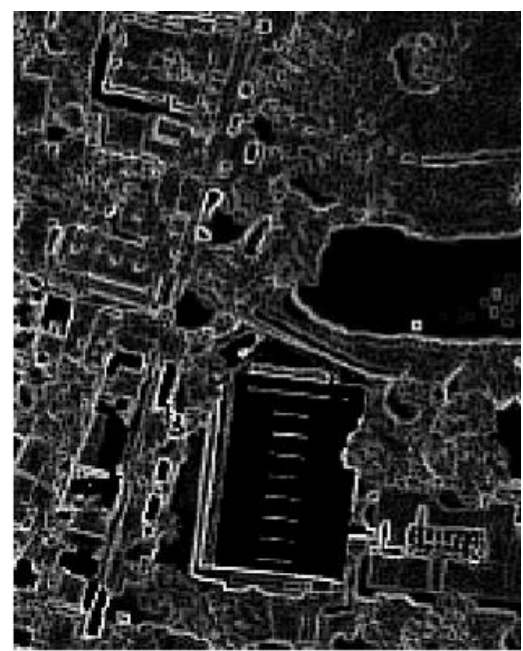

(c) $f_{6}$ (principais Componentes) 


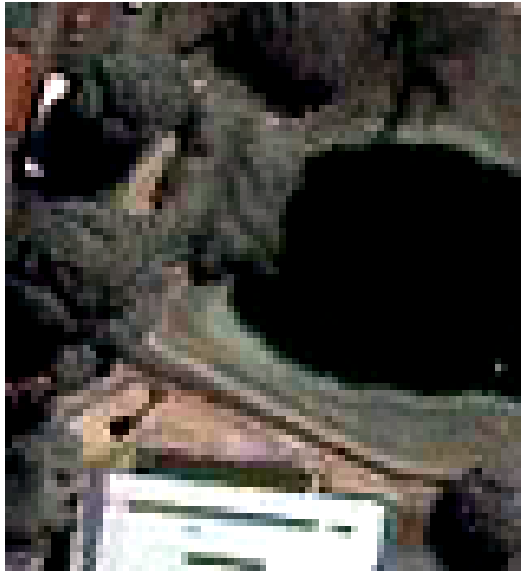

(d) Zoom da Imagem colorida $f_{1}$

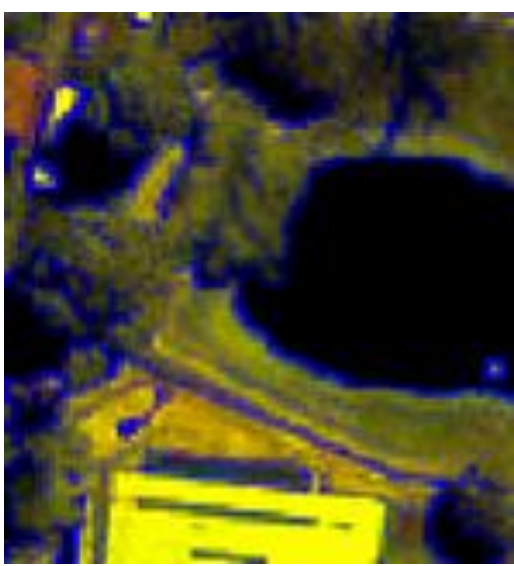

(e) Zoom da Composição RGB $f_{1, \mathrm{R}}, f_{2, \mathrm{G},} f_{6}$

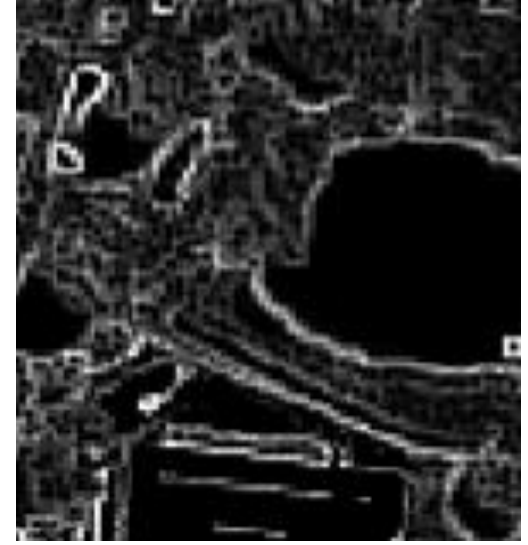

(f) Zoom da $f_{6}$ (principais Componentes)

Figura 10 - Representação da Borda externa de Principais Componentes.

\section{CONCLUSÃO}

Este trabalho apresentou as ferramentas da Morfologia Matemática para extração de bordas em imagens de alta resolução colorida. Foi usado um recorte de uma imagem do satélite IKONOS-2 fusionada, contendo o Lago do Cavouco, no entorno do Campus Joaquim Amazonas da Universidade Federal de Pernambuco (UFPE). O software SPRING foi usado no desenvolvimento da metodologia para obter a borda externa do lago. Foi apresentado uma comparação da borda para cada uma das bandas da imagem fusionada, borda obtida pela componente I da transformação IHS e PC1 das Principais Componentes. Foram calculados os histogramas, momentos, diagrama de dispersão e um perfil das imagens de borda para se comparar quantitativamente os métodos. Observou-se que a componente PC1 $\left(f_{\mathbf{6}}\right)$ gera bordas com maior contraste. Destaca-se que não foi tomado valores de limiares na obtenção das bordas de uma imagem colorida de alta resolução.

\section{AGRADECIMENTOS}

Os autores agradecem ao Departamento de Engenharia Cartográfica da UFPE e ao Conselho Nacional de Desenvolvimento Científico e Tecnológico (CNPq) pela bolsa PIBIC/CNPq de Priscilla H. O. do N., e Carla Julliane M de M. Agradecem também ao projeto FACEPE APQ-1405-1.07/12 e ao projeto FAPESP Processo FAPESP 2012/05414-5 pela imagem IKONOS2 utilizada neste trabalho e ao $\mathrm{CNPq}$ processo: 311120/2014-8.

\section{REFERÊNCIAS BIBLIOGRÁFICAS}

ANGULO, J.; SERRA, J. Color segmentation by ordered mergings, Proc. of IEEE International Conference on Image Processing (ICIP'03), IEEE,Vol. 2, Barcelona, Spain, Sept. 2003, p. 125-128.
ANGULO, J.; SERRA, J. Mathematical morphology in color spaces applied to the analysis of cartographic images, Proc. Of IEEE International Conference on Image Processing (ICIP'07), 2007.

BANON, G. J. F.; BARRERA, J. Bases da Morfologia Matemática para análise de imagens binárias. 2.ed. São José dos Campos: INPE, 1998. Disponível em: http://banon-

pc.dpi.inpe.br:1905/rep/dpi.inpe.br/banon/2000/03.15.10 33?mirror=dpi.inpe.br/banon/2001/01.11.16.21.34\&meta datarepository=dpi.inpe.br/banon/2001/01.11.16.07.22

CANDEIAS, A. L. B.; SOUSA, E. B. Aplicação da Morfologia Matemática na extração automática de curva de nível de carta topográfica. Anais do VII Simpósio Brasileiro de Sensoriamento Remoto, Salvador, 1996. p. 681-688. Disponível em:

http://marte.sid.inpe.br/col/sid.inpe.br/deise/1999/02.01.1 5.39/doc/T8.pdf

CANDEIAS, A. L.B. Aplicação da Morfologia Matemática à análise de imagens de sensoriamento remoto.1997. 187 f. Tese (Doutorado em Computação Aplicada) - Instituto Nacional de Pesquisas Aplicadas, São José dos Campos, SP,1997. Disponível em: http://mtc-

m12.sid.inpe.br/rep/dpi.inpe.br/analucia/1996/10.14.12.27 ?mirror=sid.inpe.br/banon/2001/04.06.10.52.39\&metadat arepository=sid.inpe. $\mathrm{br} / \mathrm{banon} / 2001 / 04.03 .15 .37 .23$

CANDEIAS, A. L. B.; MOURA, C. J. M. DE; NASCIMENTO, P. H. O. DO; TAVARES JR., J. R. Extração de borda: uma análise pela morfologia matemática. VIII Colóquio Brasileiro de Ciências Geodésicas, Curitiba, 2013. Disponível em: http://www.cbcg.ufpr.br/home/wpcontent/uploads/2013/11/f014_cbcg13.pdf

EOPORTALDIRECTORY (2014). IKONOS-2.

Disponível em:

https://directory.eoportal.org/web/eoportal/satellitemissions/i/ikonos-2

EVANS, A. N.; LIU, X. U. A morphological gradient approach to color edge detection. IEEE TRANSACTIONS ON IMAGE PROCESSING, 2006, 15(6): 1454-1463. 
FONSECA, L. ; NAMIKAWA, L.; CASTEJON, E.; CARVALHO, L.; PINHO, C.; PAGAMISSE, A. Image Fusion for Remote Sensing Applications, Image Fusion and Its Applications, Dr. Yufeng Zheng (Ed.), ISBN: 978953-307-182-4, InTech, DOI: 10.5772/22899. 2011.

Disponível em: http://www.intechopen.com/books/imagefusion-and-its-applications/image-fusion-for-remotesensing-applications.

SILVA, G. P.da. Uma abordagem geodésica da locação e controle dimensional de estruturas da construção civil. 2014. 174 f. Dissertação (Mestrado) Universidade Federal de Pernambuco. CTG. Programa de Pós-Graduação em Ciências Geodésicas e Tecnologias da Geoinformação, 2014. Disponível em: http://repositorio.ufpe.br/bitstream/handle/123456789/107 41/DISSERTA\%C3\%87\%C3\%830\%20Gleice\%20Pereir a\%20da\%20Silva.pdf? sequence $=1 \&$ isAllowed $=\mathrm{y}$

GONZALEZ, R. F.; WOODS, R. E. Processamento de Imagens digitais. São Paulo: Edgard Blücher, 2000.

ISHIKAWA, A. S.; SILVA, E. A.; NÓBREGA, R. A. A. Extração de Rodovias em Imagens Digitais de Alta Resolução com O Uso da Teoria de Morfologia Matemática. Revista Brasileira de Cartografia No 63/01, 2010. (ISSN 0560-4613)

LI, G.; XIAO-MING, L. Color edge detection based on mathematical morphology in HSI space, Computer and Information Application (ICCIA), 2010 International Conference on , vol., no., pp.5,8, 3-5 Dec. 2010. doi: 10.1109/ICCIA.2010.6141522

NOVO, E. M. L. Sensoriamento Remoto: princípios e aplicações.4.ed. São Paulo: Blücher, 2010.

RODRIGUES, T.G; SILVA, E. A.; LEONARDI, F. o uso de morfologia matemática na detecção de pistas em autódromo. Revista Brasileira de Cartografia No 62 Edição Especial 2, 2010. (ISSN 0560-4613), p. 337-343.

SCHOWENGERDT, R.A. Remote Sensing: Models and Methods for Image Processing. 3 edition, Academic Press, 0-12369-407-8 Diego, USA, 2007.

SERRA, J. Image analysis and mathematical morphology. London: Academic Press, 1982. 610p.

SILVA, E. A. Extração de feições cartográficas de imagens multiespectrais fundidas. São Paulo: USP, 1995. 114p. Tese (Doutorado em Engenharia) - Escola Politécnica da USP, 1995. 